\title{
Numerical Solution of two point boundary value problems by wavelet Galerkin method
}

\author{
S. Upadhyay ${ }^{1 *}$, S. Singh ${ }^{2}$, S. $\operatorname{Yadav}^{3}$, K. N. Rai ${ }^{4}$ \\ DST-CIMS, BHU, Varanasi, India \\ subabbu16@gmail.com
}

Copyright (C2014 Author. This is an open access article distributed under the Creative Commons Attribution License Creative Commons Attribution License, which permits unrestricted use, distribution, and reproduction in any medium, provided the original work is properly cited.

\begin{abstract}
In this paper, the Legendre wavelet operational matrix of integration is used to solve two point boundary value problems, in which the coefficients of the ordinary differential equation are real valued functions whose inner product with Legendre wavelet basis functions must exist. The method and convergence analysis of the Legendre wavelet is discussed. This method is applied to solve three boundary value and two moving boundary problems. In boundary value problems, we have studied the effects of condition number, elapse time and relative error on Legendre wavelet. It has been observed that the error decreases as the number of wavelet basis function increases. The condition number of square matrix of matrix equation decreases as Legendre wavelet basis function increases. The Legendre wavelet Galerkin method provides better results in lesser time, in comparison of other methods. In case of moving boundary problems the root mean square error (RMSE) for dimensionless temperature, position of moving interface and its generalized time rate are evaluated. It has been observed that the error increases as Stefan number increases.
\end{abstract}

Keywords: Legendre wavelet Galerkin method, Convergence analysis, condition numbers, elapse time, moving boundary problems

\section{Introduction}

Two point boundary value problems arise in the mathematical modelling of heat transfer in the fin [1], steady state heat transfer in biological tissues [2] and many engineering applications. These problems under most generalized boundary conditions occurs in the form

$$
\begin{aligned}
& L_{2}^{x}[y(x)]=h(x), \\
& L_{1}^{a}[y(x)]=A, \text { at } x=a, \\
& L_{1}^{b}[y(x)]=B, \text { at } x=b,
\end{aligned}
$$

where differential operator $L_{2}^{x}, L_{1}^{a}$ and $L_{1}^{b}$ defined by

$L_{2}^{x}=k(x) \frac{d^{2}}{d x^{2}}+f(x) \frac{d}{d x}+g(x)$,

$L_{1}^{a}=\alpha_{0}+\alpha_{1} \frac{d}{d x}$,

$L_{1}^{b}=\beta_{0}+\beta_{1} \frac{d}{d x}$.

$k(x), f(x), g(x)$ and $h(x)$ are real valued functions defined on the close interval $[a, b]$ and $\alpha_{0}, \alpha_{1}, \beta_{0}$ and $\beta_{1}$ are real numbers. 
The spectral representations of analytic studies of differential equations have been used in the times of Fourier. The solution of ordinary differential equations by Tau method and application of Chebyshev polynomial refers at least to time of Lanczos 1938 [3]. In the analytic study of differential equation orthogonal function and polynomials have been used by Canuto et al. 1988 [4]. If the solution functions and coefficient functions are analytic on the close interval $[a, b]$, spectral methods are very efficient and suitable [5]. If at least one of the coefficient or solution function is not analytic on the close interval $[a, b]$, the spectral method does not work very well [5], [6]. As described before, spectral (Galerkin, Tau and Pseudo-spectral) methods do not work well for this kind of problem. Subrahamanyam et al. $[17,18,19]$ applied wavelet collocation method in finite and infinite domains problems arising in engineering. F. Mohammadi et al. [7] used Galerkin method with Legendre wavelets to solve this problem with Dirichlet boundary conditions and get good results. In case of Neumann boundary value problems we can not apply a similar procedure as followed in [7]. No attention have been given to obtain the solution of a second order linear differential equation with variable coefficient under most generalized boundary conditions. The condition number of square matrix of matrix equation and elapse time for obtaining the solution by Galerkin method did not discussed in [7].

The Purpose of this work is to solve a two point boundary value problem under most generalized boundary conditions in which the coefficients of ordinary differential equation are real valued functions whose inner product with Legendre wavelet basis must exist, using Legendre wavelet Galerkin method. The method is applied to three boundary value and two moving boundary problems. The paper is organized in the following structure. In section 2 , Legendre wavelets are introduced. A general formulation of the method based on the Legendre wavelet is presented in section 3. In section 4 a brief convergence analysis is given. In section 5 numerical examples are taken for Dirichlet, Neumann and Cauchy boundary conditions. Numerical examples 5.1 is taken for elliptic differential equation with Dirichlet homogeneous boundary condition. The linear boundary value problem with Neumann boundary condition is taken in example 5.2. In example 5.3 the problem is taken for steady state temperature distribution in presence of heat source occur in [13]. The moving boundary problems with Planar melting of semi infinite medium and Planar freezing of a semi infinite medium with temperature dependent surface temperature are taken in examples 5.4 and 5.5 respectively. Section 6 represent results based discussion and its concluding remarks.

\section{Wavelets}

As per Morlet analysis, signals consist of different features in time and frequency. Their high frequency components would have shorter time duration than their low frequency components. In order to achieve a good time resolution for high frequency transients and good frequency resolution for low frequency components, Morlet (1982) first introduced the idea of wavelets as a family of functions constructed from translations and dilations of a single function called mother wavelet defined by

$\psi_{s, r}(x)=|s|^{\frac{-1}{2}} \psi\left(\frac{x-r}{s}\right), s, r \in R, s \neq 0$

where $s$ is scaling parameter, $r$ is the translation parameter and $R$ denotes the set of real numbers.

\section{1. definition}

A wavelet system for $L_{2}(R)$ is a complete orthonormal set in $L_{2}(R)$ of the form $\left\{\psi_{s, r}\right\}_{s, r \in Z}$, for some $\psi \in L_{2}(R)$,

where $Z$ denotes the set of integers and $L_{2}(R)=\left\{f: R \rightarrow C: \int_{R}|f(x)|^{2} d x<\infty\right\}$. The functions $\psi_{s, r}$ are called wavelets. The function $\psi$ is called the mother wavelet.

\section{2. $\quad$ Legendre wavelets}

The Legendre wavelets, $\psi_{n, m}(x)=\psi(k, \hat{n}, m, x)$ have four arguments

$k=1,2,3, \ldots$,

$n=1,2, \ldots, 2^{k-1}$,

$\hat{n}=2 n-1$,

$m$ is the order of Legendre polynomial and $x$ is the normalized time. The Legendre wavelets are defined on the closed interval $[0,1]$ by

$\psi_{n, m}(x)= \begin{cases}\sqrt{(m+1 / 2)} 2^{k / 2} P_{m}\left(2^{k} x-\hat{n}\right) & , \quad \frac{\hat{n}-1}{2^{k}} \leq x \leq \frac{\hat{n}+1}{2^{k}} \\ 0 & , \quad \text { otherwise }\end{cases}$ 
where the dilation parameter is $s=2^{-k}$ and the translation parameter is $r=\hat{n} 2^{-k} . P_{m}(x)$ denotes the Legendre polynomial of order $m, m=0,1, \ldots, M-1$, which are orthogonal with respect to the weight function $w(x)=1$ on the interval $[-1,1]$, and satisfy the following recursive formula

$P_{0}(x)=1$,

$P_{1}(x)=x$,

$P_{m+1}(x)=\frac{2 m+1}{m+1} x P_{m}(x)-\frac{m}{m+1} P_{m-1}(x)$.

\subsection{Fourier approximation:}

A function defined in the domain [a, b] may be expressed as

$f(x)=\sum_{n=1}^{\infty} \sum_{m=0}^{\infty} c_{n, m} \psi_{n, m}(x)$,

where $c_{n, m}=<f(x), \psi_{n, m}(x)>$ in which $<,>$ denotes the inner product. If the infinite series in (6) is truncated, then it can be written as

$f(x) \approx \sum_{n=1}^{2^{k-1}} \sum_{m=0}^{M-1} c_{n, m} \psi_{n, m}(x)=C^{T} \psi(x)$

where $C$ and $\psi(x)$ are column matrices of order $2^{k-1} M \times 1$, given by

$C=\left[c_{10}, c_{11}, \ldots, c_{1 M-1}, c_{20}, c_{21}, \ldots, c_{2 M-1}, \ldots, c_{2^{k-1} 0}, c_{2^{k-1} 1}, \ldots, c_{2^{k-1} M-1}\right]^{T}$

$\psi(x)=\left[\psi_{10}(x), \psi_{11}(x), \ldots, \psi_{1 M-1}(x), \psi_{20}(x), \ldots, \psi_{2 M-1}(x), \ldots, \psi_{2^{k-1} 0}(x), \psi_{2^{k-1} 1}(x), \ldots, \psi_{2^{k-1} M-1}(x)\right]^{T}$.

\subsection{Property of the product of two Legendre wavelets}

If $E$ is a given wavelets vector then we have the property

$E^{T} \psi \psi^{T}=\psi^{T} \hat{E}$

where $\hat{E}$ is $2^{k-1} M \times 2^{k-1} M$ matrix depending on the wavelet vector $E$. The technique of calculation of $P$ and $\hat{E}$ are illustrated in [7] and [10].

\subsection{Operational matrix of integration}

The integration of the wavelets $\psi(x)$ can be obtained as

$$
\int_{0}^{x} \psi(s) d s=P \psi(x)
$$

where $P$ is a $2^{k-1} M \times 2^{k-1} M$ operational matrix of integration defined by

$$
P=\frac{1}{2^{k}}\left(\begin{array}{ccccc}
L & F & F & \cdots & F \\
0 & L & F & \cdots & F \\
0 & 0 & L & \cdots & F \\
\vdots & \vdots & \vdots & \ddots & \vdots \\
0 & 0 & 0 & \cdots & F \\
0 & 0 & 0 & \cdots & F
\end{array}\right)
$$

$F$ and $L$ are $M \times M$ matrices given by

$$
F=\left(\begin{array}{cccc}
2 & 0 & \cdots & 0 \\
0 & 0 & \cdots & 0 \\
\vdots & \vdots & \ddots & \vdots \\
0 & 0 & \cdots & 0
\end{array}\right)
$$


and

$$
L=\left(\begin{array}{cccccccc}
1 & \frac{1}{\sqrt{3}} & 0 & 0 & \cdots & 0 & 0 & 0 \\
\frac{-1}{\sqrt{3}} & 0 & \frac{1}{\sqrt{15}} & 0 & \cdots & 0 & 0 & 0 \\
0 & \frac{-1}{\sqrt{15}} & 0 & \frac{1}{\sqrt{35}} & \cdots & 0 & 0 & 0 \\
0 & 0 & \frac{-1}{\sqrt{35}} & 0 & \cdots & 0 & 0 & 0 \\
\vdots & \vdots & \vdots & \vdots & \ddots & \vdots & \vdots & \vdots \\
0 & 0 & 0 & 0 & \cdots & \frac{1}{\sqrt{(2 M-3)(2 M-5)}} & 0 & \frac{1}{\sqrt{(2 M-3)(2 M-1)}} \\
0 & 0 & 0 & 0 & \cdots & 0 & \frac{-1}{\sqrt{(2 M-1)(2 M-3)}} & 0
\end{array}\right) \text { respectively. }
$$

\section{Legendre wavelet Galerkin Method}

We assume that the unknown function $y^{\prime \prime}(x)$ is

$y^{\prime \prime}(x)=C^{T} \psi(x)$,

where $C$ is an unknown vector and $\psi(x)$ is a vector defined in Eqs. (8) and (9) respectively. Integrating Rq. (13) from 0 to $x$ and using Eq. (11), we get

$y^{\prime}(x)=y^{\prime}(0) d^{T} \psi(x)+C^{T} P \psi(x)$,

where $d$ is the vector coefficient of the unit step function, we can also express $k(x), f(x), g(x), h(x)$ as

$k(x)=K^{T} \psi(x), f(x)=F^{T} \psi(x), g(x)=G^{T} \psi(x), h(x)=H^{T} \psi(x)$.

Again integrating Eq. (14) from 0 to $x$ and using Eq. (11), we get

$y(x)=y(0)+y^{\prime}(0) d^{T} P \psi(x)+C^{T} P^{2} \psi(x)$.

Put $x=a$ in Eqs. (14) and (16), we get

$$
\begin{aligned}
& y^{\prime}(a)=y^{\prime}(0) d^{T} \psi(a)+C^{T} P \psi(a), \\
& y(a)=y(0)+y^{\prime}(0) d^{T} P \psi(a)+C^{T} P^{2} \psi(a) .
\end{aligned}
$$

Again putting $x=b$ in Eqs. (14) and (16), we get

$$
\begin{aligned}
& y^{\prime}(b)=y^{\prime}(0) d^{T} \psi(b)+C^{T} P \psi(b), \\
& y(b)=y(0)+y^{\prime}(0) d^{T} P \psi(b)+C^{T} P^{2} \psi(b) .
\end{aligned}
$$

Putting $y^{\prime}(a), y(a)$ from Eqs. (17), (18) and $y^{\prime}(b), y(b)$ from Eqs. (19), (20) Eqs. (2), (3), we get

$$
\begin{aligned}
& \alpha_{0} y(0)+\left(\alpha_{0} d^{T} P \psi(a)+\alpha_{1}\right) y^{\prime}(0)=A-\alpha_{0} C^{T} P^{2} \psi(a)-\alpha_{1} C^{T} P \psi(a), \\
& \beta_{0} y(0)+\left(\beta_{0} d^{T} P \psi(b)+\beta_{1}\right) y^{\prime}(0)=B-\beta_{0} C^{T} P^{2} \psi(b)-\beta_{1} C^{T} P \psi(b) .
\end{aligned}
$$

Solving Eqs. (21) and (22), we have two different cases.

Case $1 \alpha_{0} \neq 0, \beta_{0}^{2}+\beta_{1}^{2} \neq 0$.

Multiplying $\beta_{0}$ in Eq. (21) and $\alpha_{0}$ in Eq. (22) and subtracting it, we get

$y^{\prime}(0)=\frac{R_{0}}{Q_{0}}$,

where

$$
\begin{aligned}
& R_{0}=\beta_{0} A-\alpha_{0} B+\alpha_{0} \beta_{0} C^{T} P^{2}(\psi(b)-\psi(a))+\alpha_{0} \beta_{1} C^{T} P \psi(b)-\alpha_{1} \beta_{0} C^{T} P \psi(a) \\
& Q_{0}=\alpha_{1} \beta_{0}-\alpha_{0} \beta_{1}-\alpha_{0} \beta_{0} d^{T} P(\psi(b)-\psi(a)) .
\end{aligned}
$$

Put $y^{\prime}(0)$ in Eq. (21), we get

$y(0)=\frac{a-\alpha_{0} C^{T} P^{2} \psi(a)-\alpha_{1} C^{T} P \psi(a)-\left(\alpha_{0} d^{T} P \psi(a)+\alpha_{1}\right) \frac{R_{0}}{Q_{0}}}{\alpha_{0} .}$ 
From Eqs. (14) and (23) we have

$y^{\prime}(x)=\frac{R_{0}}{Q_{0}} d^{T} \psi(x)+C^{T} P \psi(x)$.

From Eqs. (16), (23) and (24) we have

$y(x)=\frac{A-\alpha_{0} C^{T} P^{2} \psi(a)-\alpha_{1} C^{T} P \psi(a)-\left(\alpha_{0} d^{T} P \psi(a)+\alpha_{1}\right) \frac{R_{0}}{Q_{0}}}{\alpha_{0}}+\frac{R_{0}}{Q_{0}} d^{T} P \psi(x)+C^{T} P^{2} \psi(x)$.

Using Eqs. (13), (15), (25) and (26) in Eq. (1), taking transpose and applying Eq. (10), we get the system of linear equations given by

$\left(\hat{K}+\hat{F} P^{T}-\hat{G} d \psi^{T}(a) P^{2 T}-\frac{\alpha_{1}}{\alpha_{0}} \hat{G} d \psi^{T}(a) P^{T}+\hat{G} P^{2 T}\right) C+\left(\hat{F} d-\frac{\alpha_{1}}{\alpha_{0}} \hat{G} d+\hat{G} P^{T} d\right) \frac{R_{0}^{T}}{Q_{0}}-\hat{G} d \frac{R_{0}^{T}}{Q_{0}} \psi^{T}(a) P^{T} d=H-\frac{\alpha_{A}}{\alpha_{0}} \hat{G} d$.

Case $2 \alpha_{1} \neq 0, \alpha_{0}^{2}+\beta_{0}^{2} \neq 0$ and $\alpha_{1}^{2}+\beta_{1}^{2} \neq 0$. The Eq. (21) becomes of the form

$y^{\prime}(0)=\frac{A-\alpha_{0} C^{T} P^{2} \psi(a)-\alpha_{1} C^{T} P \psi(a)-\alpha_{0} y(0)}{\alpha_{0} d^{T} P \psi(a)+\alpha_{1}}$.

Putting $y^{\prime}(0)$, from Eq. (28) in Eq. (22) we get

$y(0)=\frac{U}{V}$,

where

$U=B-\beta_{0} C^{T} P^{2} \psi(b)-\beta_{1} C^{T} P \psi(b)-\left(\beta_{0} d^{T} P \psi(b)+\beta_{1}\right) \frac{A-\alpha_{0} C^{T} P^{2} \psi(a)-\alpha_{1} C^{T} P \psi(a)}{\alpha_{0} d^{T} P \psi(a)+\alpha_{1}}$,

and

$V=\beta_{0}-\frac{\left(\beta_{0} d^{T} P \psi(b)+\beta_{1}\right)}{\left(\alpha_{0} d^{T} P \psi(a)+\alpha_{1}\right)} \alpha_{0}$

From Eqs. (28) and (29), we get

$y^{\prime}(0)=\frac{A-\alpha_{0} C^{T} P^{2} \psi(a)-\alpha_{1} C^{T} P \psi(a)-\alpha_{0} \frac{U}{V}}{\alpha_{0} d^{T} P \psi(a)+\alpha_{1}}$.

From Eqs. (14) and (30), we get

$y^{\prime}(x)=\left\{\frac{A-\alpha_{0} C^{T} P^{2} \psi(a)-\alpha_{1} C^{T} P \psi(a)-\alpha_{0} \frac{U}{V}}{\alpha_{0} d^{T} P \psi(a)+\alpha_{1}}\right\} d^{T} \psi(x)+C^{T} P \psi(x)$.

From Eqs. (15), (29) and (30), we get

$\left.y(x)=\frac{U}{V}+\frac{A-\alpha_{0} C^{T} P^{2} \psi(a)-\alpha_{1} C^{T} P \psi(a)-\alpha_{0} \frac{U}{V}}{\alpha_{0} d^{T} P \psi(a)+\alpha_{1}}\right\} d^{T} \psi(x)+C^{T} P^{2} \psi(x)$.

Applying similar process to those given in case 1, we get the system of linear equation given by

$$
\begin{gathered}
\left(\hat{K}+\hat{F} P^{T} \hat{G} P^{2 T}\right) C+(\hat{F} d+\hat{G} P T d)\left(\frac{A-\alpha_{0} C^{T} P^{2} \psi(a)-\alpha_{1} C^{T} P \psi(a)-\alpha_{0} \frac{U}{V}}{\alpha_{0} d^{T} P \psi(a)+\alpha_{1}}\right)^{T} \\
+\hat{G} d \frac{U^{T}}{V}=H .
\end{gathered}
$$

The Eqs. (27) and (33) represents the system of linear equations for unknown vector $C$. These equations can be solved for vector $C$. After finding the unknown vector $C$, we can get the approximation solution of Eq. (1) by 
inserting $C$ into Eqs. (26) and (32) for different boundary conditions.

Case $3 \alpha_{0}=\beta_{0}=a=0, \alpha_{1} \neq 0$ and $\beta_{1} \neq 0$. In this case we cannot use similar procedure as described in Case 1 -2, because unknown $y(0)$ can not determined by Eq. (14) or (16) with given boundary conditions. In this situation, we take $y(0)=\gamma$ as a constant and solving Eq. (1) as an initial value problem with initial conditions $y(0)=\gamma$ and $y^{\prime}(0)=\frac{A}{\alpha_{1}}$, then Eqs. (14) and (16) come out to be

$$
\begin{array}{r}
y^{\prime}(x)=\frac{A}{\alpha_{1}} d^{T} \psi(x)+C^{T} P \psi(x), \\
y(x)=\gamma d^{T} \psi(x)+\frac{A}{\alpha_{1}} d^{T} P \psi(x)+C^{T} P^{2} \psi(x) .
\end{array}
$$

Using Eqs. (13), (15), (34) and (35) in Eq. (1), we have

$$
K^{T} \psi(x) C^{T} \psi(x)+F^{T} \psi(x)\left(\frac{A}{\alpha_{1}} d^{T} \psi(x)+C^{T} P \psi(x)\right)+G^{T} \psi(x)\left(\gamma+\frac{A}{\alpha_{1}} d^{T} P \psi(x)+C^{T} P^{2} \psi(x)\right)=H^{T} \psi .
$$

Taking transpose both sides of Eq. (36) and using Eq. (10), we have

$$
\left(\hat{K}+\hat{F} P^{T}+\hat{G} P^{2 T}\right) C=H-\gamma \hat{G} d-\frac{A}{\alpha_{1}}\left(\hat{G} P^{T} d+\hat{F} d\right) .
$$

The Eqs. (37) can be solved for vector $C$. After finding the unknown vector $C$, we can get the approximation solution in terms of $\gamma$ and $x$ by inserting $C$ into Eq. (15). For eliminating $\gamma$, we will use second boundary condition $y^{\prime}(b)=\frac{B}{\beta_{1}}$.

\section{Convergence Analysis}

To compute approximate error, we introduce a subspace $V_{2^{k-1} M}$ of $L_{2}[0,1]$ generated by an orthonormal set

$$
\left\{\psi_{n, m}(x): n=1,2, \ldots, 2^{k-1}, m=0,1, \ldots M-1 .\right\}
$$

have dimension $2^{k-1} M$, where $\psi_{n, m}(x)$ defined in section 2.2 and $k$ is a non zero fixed positive integer.

Theorem 4.1. Let $y(x)$ be a real valued function defined on $[0,1]$ and its $m t h$ derivative $y^{(m)}(x)$ bounded on $[0,1]$, the following inequality holds

where

$$
e(m, y) \leq \frac{K}{m !},
$$

$$
\begin{gathered}
e(m, y)=\|y(x)-\check{y}(x)\|_{L_{2}[0,1]}, \\
K=\sup \left\{y^{(m)}(x): x \in[0,1]\right\},
\end{gathered}
$$

and $\check{y}(x)$ denotes the Legendre wavelet solution of boundary value problem (1) - (3).

Proof: The approximate solution of boundary value problem (1) - (3) can be written in the form

$\check{y}(x)=\sum_{n=1}^{2^{k-1}} \sum_{m=0}^{M-1} d_{n, m} \psi_{n, m}(x)$,

with restriction that $\check{y}(x)$ is a polynomial of degree $m$ that approximate $y^{*}(x)$ with minimum mean square error, where $\psi_{n, m}(x)$ is the Legendre wavelet of the approximation subspace $V_{2^{k-1} M}$ of $L_{2}[0,1]$ and $d_{n, m}$ denotes the coefficients of Legendre wavelet basis functions series.

$\left\|y(x)-\sum_{n=1}^{2^{k-1}} \sum_{m=0}^{M-1} d_{n, m} \psi_{n, m}(x)\right\|_{L_{2}[0,1]}^{2} \leq \int_{0}^{1}\left[y(x)-y^{*}(x)\right]^{2} d x$,

where $y^{*}(x)$ is interpolating polynomials of $\mathrm{y}(\mathrm{x})$ and we have used well known maximum error bound for interpolation.

$$
\begin{aligned}
& \int_{0}^{1}\left[y(x)-y^{*}(x)\right]^{2} d x \leq \int_{0}^{1}\left[\frac{1}{m ! 2^{m k}} \operatorname{Sup}\left\{y^{(m)}(x): x \in[0,1]\right\}\right]^{2} d x \\
& \leq\left[\frac{K}{m ! 2^{m k}}\right]^{2}
\end{aligned}
$$


$\leq\left[\frac{K}{m !}\right]^{2}$

From (38) and (39), we have required result.

$e(m, y) \leq \frac{K}{m !}$

Equation (40), shows that $e(m, y)$ approaches to zero as $m$ tend to infinity.

Theorem 4.2. Let $E(x)$ be real valued function defined on $L_{2}[0,1]$ and $\breve{y}(x)$ be a Legendre wavelets solution of (1) - (3). Then there exist constants $D$ and $K \geq 0$ such that $D=-\left(y(0)+y^{\prime}(0) d^{T} P d+C^{T} P^{2} d\right)$, whenever $D=\sup \{E(x): x \in[0,1]\}$, and $K=-\left(\int_{0}^{1} \check{y}(x) d x\right)^{2}+\int_{0}^{1} \check{y}^{2}(x) d x$.

Proof: Since

$$
\begin{aligned}
& <\check{y}(x)+E(x), \check{y}(x)+E(x)>=\int_{0}^{1} \check{y}^{2}(x) d x+2 \int_{0}^{1} \check{y}(x) E(x) d x+\int_{0}^{1} E^{2}(x) d x \\
& \leq D^{2}+2 D \int_{0}^{1} \check{y}(x) d x+\int_{0}^{1} \check{y}^{2}(x) d x,
\end{aligned}
$$

then there is a constant $K \geq 0$ such that

$D^{2}+2 D \int_{0}^{1} \check{y}(x) d x+\int_{0}^{1} \check{y}^{2}(x) d x-K=0$.

The root of above quadratic equation in $D$ is

$D=-\int_{0}^{1} \check{y}(x) d x$,

whenever

$K=-\left(\int_{0}^{1} \check{y}(x) d x\right)^{2}+\int_{0}^{1} \check{y}^{2}(x) d x$.

Obtained $D$ and from Eq. (16), we get

$D=-y(0)-y^{\prime}(0) d^{T} P d-C^{T} P^{2} d$.

\section{Numerical performances}

To show the efficiency of the Legendre wavelet Galerkin method for different $M$ and $k$ (LWGM $(M, k)$ ), we consider three boundary value problems (Examples 5.1 - 5.3) and two moving boundary problems (Examples 5.4 - 5.5). We solved following examples by using Legendre wavelet Galerkin method describe in section 3. The computations associated with the examples are performed by MATLAB with programming, see in[15].

\subsection{Dirichlet homogeneous boundary condition}

Example 5.1: Lotfi and Mahdiani [9] used wavelet Galerkin method taking Coiflet wavelets to solve elliptic boundary value problem with Dirichlet homogeneous boundary condition,

$-y^{\prime \prime}(x)+\pi^{2} y(x)=2 \pi^{2} \sin (\pi x), x \in(0,1)$ 
$y(1)=y(0)=0$.

We solve this boundary value problem using the LWGM $(M, k)$. The vector $C$ is computed by solving the system of linear Eq. (27). The condition number of square matrices of matrix Eq. (27) for different $(M, 1)$ are given in Table 1. The comparative study of absolute errors in LWGM solution and in Coiflet [9] solution are given in Table 2 .

\subsection{Neumann boundary value problem}

Example 5.2: To solve linear boundary value problem with Neumann boundary conditions, Rahmani et al.[14] used Galerkin method with Hermite polynomials as a basis functions.

$$
\begin{aligned}
& y^{\prime \prime}(x)+y(x)=\cos (x), x \in[0,5], \\
& y^{\prime}(0)=y^{\prime}(5)=0 .
\end{aligned}
$$

Under transform

$x=t / 5$,

the Eqs. (41) and (42) reduced in the form

$$
\begin{aligned}
& u^{\prime \prime}(x)+25 u(x)=25 \cos (5 x), x \in[0,1], \\
& u^{\prime}(0)=u^{\prime}(1)=0 .
\end{aligned}
$$

The exact solution of Eqs. (43) - (44) is

$u(x)=\frac{\sin (5)+10 \cos (5)}{4 \sin (5)} \cos (5 x)+(5 / 2) x \sin (5 x)+(1 / 4) \cos (5 x)$.

The solution obtained by present method is

$u(x)=\gamma d^{T} \psi+C^{T} P^{2} \psi(x)$,

where $C$ is determined by

$\left(25 P^{2 T}+I\right) C=H-25 \gamma d$

and $\gamma$ is determined by using the boundary condition at $x=1$. The technique for the solution of such types of Neumann boundary value problem by LWGM described in section 3 (Case 3 ).

The numerical solutions of (43) - (44) by present method and Galerkin method with Hermite polynomials (HGM) are given in Table 3 .

\subsection{Cauchy boundary value problem}

Example 5.3: Mathematical formulation of steady state temperature distribution in presence of heat source in solid cable of unit length who's one end insulated and other kept at fixed temperature occur in form [13]

$$
\begin{aligned}
& -y^{\prime \prime}(x)=h(x), x \in[0,1], \\
& y^{\prime}(0)=y(1)=0,
\end{aligned}
$$

where $y(x)$ is the time-independent temperature and $h(x)$ is the heat source. Stpiczynski solved this problem by finite difference scheme implemented in Fermi the next generation CUDA computing architecture and studied the elapse time and relative error, given in reference [13]. The solution of the problem (45) - (46) by present method comes out to be

$$
y(x)=C^{T} P^{2} \psi(x)-C^{T} P^{2} \psi(1),
$$

where $C$ are evaluated from the system of linear equations

$I C=-H$,

and $I$ is $2^{k-1} M \times 2^{k-1} M$ identity matrix.

The numerical computation for $h(x)=\frac{\pi^{2}}{4} \cos (\pi x / 2)$, is studied. The sparse matrix $I$ has condition number 1.0 because of this the system $I C=-H$ becomes well conditioned. The relative error for different number of Legendre wavelet basis functions is given in Table 4. The execution time (in seconds) during in the process to determine $C$ and $y(x)$ are given in Table 5 . 


\subsection{Planar melting of semi infinite medium}

Example 5.4: Let us consider a semi-infinite solid initially at a temperature $T_{s}$ which is slightly below the melting temperature $T_{m}$ of the solid. At the time $t=0$, the surface is kept at temperature $T_{0}>T_{m}$. The solid melts and solid liquid interface progresses into the interior of solid with time. The solid phase being at a constant temperature throughout, but the temperature in the liquid phase is unknown. Hence, the problem is a one phase problem. The dynamics of melting can be described by the following equations in dimensionless form

$\frac{\partial \theta}{\partial F_{o}}=\frac{\partial^{2} \theta}{\partial y^{2}}, 0<y<x_{f}, F_{o}>0$

The associated boundary conditions are

$$
\begin{aligned}
& \theta\left(0, F_{o}\right)=1, \\
& \theta\left(x_{f}, F_{o}\right)=0,
\end{aligned}
$$

and the interface condition is

$\frac{\partial \theta\left(x_{f}, F_{o}\right)}{\partial F_{o}}+\frac{1}{S} \frac{\partial x_{f}}{\partial F_{o}}=0$.

Under the transformation

$x=\frac{y}{x_{f}}$,

$x_{f}=\frac{2 \mu}{\left.2 \sqrt{(} F_{o}\right)}$,

the system of Eqs. (47) - (50) reduces to

$$
\begin{aligned}
& \theta^{\prime \prime}(x)+2 \mu^{2} x \theta^{\prime}(x)=0, \\
& \theta(0)=1, \\
& \theta(1)=0, \\
& \theta^{\prime}(1)+\frac{2 \mu^{2}}{S}=0 .
\end{aligned}
$$

The exact solution of Eqs. (51) - (54) occur in the form

$\theta(x)=1-\frac{\operatorname{erf}(\mu x)}{\operatorname{erf}(\mu)}$.

Using (55) in (54), we obtain a non linear algebraic equation. To solve equation (51) with boundary conditions (52) - (53), using LWGM $(M, k)$. The vector $C$ is computed by solving the system of linear equation $(27)$. We get $\theta(x)$ from equation (26). Substituting the value of $\theta(x)$ in equation (54), we obtain a non linear algebraic equation in $\mu$.

To obtain $\mu$, we solve these non-linear algebraic equations obtained in exact method and in Legendre Wavelet Galerkin Method using Newton-Raphson method up to ten iterations. We obtain $\mu$ for different S. A comparison of $\theta(x)$ obtained by exact method and $\operatorname{LWGM}(7,1)$ is given in Table 6 . The comparison of the value of $x_{f}$ and $\frac{d x_{f}}{d F_{o}}$ for different $F_{o}$ obtained by exact method and Legendre wavelet Galerkin method are given in Tables 7 and 8 . The RMSE of $\theta(x)$ are $2.5584 \times 10^{-6}, 8.5067 \times 10^{-6}, 3.4727 \times 10^{-5}$, RMSE of $x_{f}$ are $0,0,1.44 \times 10^{-4}$ and RMSE of $\frac{d x_{f}}{d F_{o}}$ are $0,0,1.89 \times 10^{-4}$ for $S=1,3,5$. respectively.

\subsection{Planar freezing of semi infinite medium with time dependent surface tempera- ture}

Example 5.5;: A line heat sink placed at origin of the planner coordinate in a medium consisting of liquid initially at temperature $T_{f}$. At time $t=0$ the surface temperature of heat sink decreases linearly with time i.e.

$T(0, t)=T_{f}-b t$. 
The freezing process begins and freezing zone grows outward. The temperature distribution in the solid region in dimensionless form is governed by the following equations

$$
\begin{array}{r}
\frac{\partial \theta}{\partial F_{o}}=\frac{\partial^{2} \theta}{\partial y^{2}}-\frac{\theta}{F_{o}}, \\
\theta\left(0, F_{o}\right)=1, \\
\theta^{\prime}\left(x_{f}, F_{o}\right)=0, \\
\frac{\partial \theta\left(x_{f}, F_{o}\right)}{\partial F_{o}}+\frac{1}{S} \frac{\partial x_{f}}{\partial F_{o}}=0 .
\end{array}
$$

Under the transformation

$x=\frac{y}{2 \sqrt{\left(F_{o}\right)}}, \mu=\frac{x_{f}}{2 \sqrt{\left(F_{o}\right)}}$.

The system of equations (57) - (60) reduce to

$$
\begin{aligned}
\theta^{\prime \prime}(x)+2 x \theta^{\prime}(x)-4 \theta(x) & =0, \\
\theta(0) & =1, \\
\theta(\mu) & =0, \\
\theta^{\prime}(\mu)+\frac{2 \mu}{S} & =0 .
\end{aligned}
$$

The exact solution of equations (61) - (64) occur in the form

$$
\theta(x)=\left(1+2 x^{2}\right) \frac{\int_{x}^{\mu} \exp \left(-2 \log \left(s^{2}+\frac{1}{2}\right)+s^{2}\right) d s}{\int_{0}^{\mu} \exp \left(-2 \log \left(x^{2}+\frac{1}{2}\right)+x^{2}\right) d x} .
$$

Using (65) in (64), we obtain a non linear algebraic equation in $\mu$.

To solve equation (61) with boundary conditions (62) - (63), using LWGM $(M, k)$. The vector $\mathrm{C}$ is computed by solving the system of linear equation (27). We get $\theta(x)$ from (26). Substituting the value of value $\theta(x)$ in equation (64), we obtain a non linear algebraic equation in $\mu$.

To obtain $\mu$, we solve these non-linear algebraic equations obtained in exact method and LWGM, using Newton Raphson method up to ten iterations. We obtain $\mu$ for different S. The variation of dimensionless temperature $\theta(x)$ obtained by LWGM (6.1), for different $S$ is given in Figure 1. The value of $x_{f}$ and $\frac{d x_{f}}{d F_{o}}$ for different $F_{o}$ obtained by exact method and LWGM are given in Table 9 and 10. The value of $x_{f}$ and $\frac{d x_{f}}{d F_{o}}$ for different $F_{o}$ obtained by LWGM (6.1), for $\mathrm{S}=4,6,8,10$ and 12 represented in Figure 2 and 3. The RMSE of $x_{f}$ are $0,0,1.84 \times 10^{-4}$ and RMSE of $\frac{x_{f}}{d F_{o}}$ are $0,0,2.46 \times 10^{-4}$ for $\mathrm{S}=1,3,5$ respectively.

\section{Results and Discussion}

In the section 3 we have used Legendre wavelet Galerkin method in two point boundary value problem under most generalized boundary condition and discussed its particular cases. In Case 3, we described a new approach for solving Neumann boundary value problems in sense of Legendre wavelet Galerkin method. From Theorem 4.1, we conclude that the $L_{2}$ norm of the error in LWGM solution approaches to zero as $m$ tends to infinity. It is evident from Theorem 4.2 that the maximum error in LWGM solution is bounded so LWGM is stable.

The effects of Legendre wavelets on condition number, computational complexity, time complexity, relative error in LWGM solution and application of LWGM in moving boundary problem are given in particular examples 5.1 5.5. The results based discussion on Examples 5.1 - 5.5 given as follows:

- In Example 5.1, we have solved elliptic boundary value problem with Dirichlet homogeneous boundary conditions using present method and compared with exact solution and Coiflets wavelets Galerkin solution [9]. From Table 1, we observed that the condition number of square matrix of matrix equation decreases as number of Legendre wavelet basis functions increases and system becomes well conditioned. Urban [12] used Galerkin method in two point boundary value problem with basis functions as hat MRA generated by hat function. The stiffness matrix form tridiagonal matrix have condition number $o\left(2^{2} j\right)$. Thus condition number tends to 
infinity as $\mathrm{j}$ tends to infinity. In finite difference scheme [8] for two point boundary value problem, the condition number is $o\left(N^{2}\right)$ i.e. tends to infinity as $N$ tends to infinity and so the system is ill conditioned. Because of these the hat function Galerkin method [12] and finite difference scheme [8] for two point boundary value problem have more computational complexity but LWGM have no computational complexity. From Table 2, we observe that the LWCM provides better accuracy that Coiflet wavelet Galerkin method. The absolute error in LWGM solution decreases and in CWGM solution increases as $x$ increases on the domain $[0.7,1]$. The Coiflets are orthogonal wavelets for which $\psi$ ( Mother wavelet ) has several vanishing moments, and for which $\phi$ ( Father wavelet ) also has several vanishing moments. Coiflets first appeared in [16]. They are named after Ronald Coifman, who requested such wavelets from Ingrid Daubechies. In Daubechiess original approach, the number of vanishing moments for $\phi$ and $\psi$ was taken to be equal. The Daubechies wavelet construction had the orthogonality relation built in. The coiflet construction does not. This means we have to enforce orthogonality by imposing conditions. The resulting coefficients are listed in appendix A of [16]. The two different Coiflets of length 6 with support $[2,3]$ can be find and the coefficients of scaling function are obtained by iterative process because of this, Legendre wavelets Galerkin method provides better results than Coiflet wavelet Galerkin method.

- Linear boundary value problem with Neumann boundary condition are taken in Example 5.2. We solved this problem by LWGM and compared with Galerkin Method with Hermite polynomials as a basis function. In solution of linear boundary value problem with Neumann boundary condition, the LWGM $(9,1)$ provides better results in comparison to Galerkin method with Hermite polynomials up to 12 degree as given in Table 3 . The absolute error in HWM solution increases and in LWGM solution decreases as $x$ increases on the domain $[0.5,1]$. The maximum absolute error in present method is $10^{-4}$ which is lesser than Galerkin method with Hermite polynomials [14]. The Hermite polynomials taken in [14] are orthogonal in $R$ with weight function $\exp \left(-x^{2}\right)$ because of this the Legendre wavelet Galerkin method provides better result than Galerkin method with Hermite polynomials.

- It is clear from Tables 4 - 5 that the Legendre Wavelet Galerkin Method provides better results in lesser time, comparison to finite difference scheme implemented in Fermi the next generation CUDA computing architecture [13] and relative error decreases as number of Legendre wavelet basis functions increases.

- In Example 5.4, we consider planner melting of semi infinite medium. It is clear from Table 6 - 8, that the results obtained by present method are exactly the same as that obtained by exact method. The RMSE for dimensionless temperature, position of moving interface and its generalized time rate are increases as Stephan number $S$ increases.

- The Planner freezing of semi infinite medium due to a line heat sink whose surface temperature decreases linearly with time is studied in the example 5.5. The results thus obtained are compared with exact solution and are in good agreement. In exact solution the computation is difficult and truncation error is large. Because of this the results obtained by present method are more accurate than that of exact method. In LWGM solution, the RMSE for position of moving interface and its generalized time rate are increases as Stefan number $S$ increases.

- It is evident from Tables 9 - 10 that error increases as $\mathrm{S}$ increases. Therefore, higher value of Stefan number requires higher number of Legendre wavelet basis functions.

- The results are shown in Figures 1 - 3 are important for physical aspects. From Figures 1 - 3, we conclude that the dimensionless temperature decreases as $x$ increases and Stafen number decreases. The position of moving interface and its generalized time rate increases as Stafen number increases.

\subsection{Conclusion}

We have solved two point boundary value problem under most generalized condition and discussed its particular cases 1, 2, 3, using Legendre wavelet Galerkin method. In Case 3, we described a new approach for solving Neumann boundary value problems in sense of Legendre wavelet Galerkin method. The present method is easy to understand, no any complexity in computations and easy to implementable on MATLAB. From Theorem 4.1 and 4.2 ; we conclude that $L_{2}$ norm of error tends to zero as $m$ tend to infinity and maximum error on the closed interval $[0,1]$ is bounded. From above results and discussion we conclude that the method provides good result and error can be minimized and approaches to zero by increasing the number of Legendre wavelet basis functions. The condition numbers decreases as Legendre wavelet basis functions increases. The relative error and taken elapse time 
Table 1: The effect of Legendre wavelets on condition number (CN)

\begin{tabular}{lccccccc}
\hline $\mathrm{M}$ & 3 & 4 & 5 & 7 & 9 & 10 & 11 \\
\hline $\mathrm{CN}$ & 3.97200 & 2.03970 & 2.00510 & 2.0019 & 2.00090 & 1.95620 & 1.95593 \\
\hline
\end{tabular}

Table 2: Absolute error (AE) in Coiflets solution and LWGM solution.

\begin{tabular}{lcc}
\hline $\mathrm{x}$ & AE in Coiflets solution & AE in LWGM solution \\
\hline 0.0 & 0 & $1.3090 \times 10^{-7}$ \\
0.1 & $1.5199 \times 10^{-4}$ & $3.5999 \times 10^{-8}$ \\
0.2 & $2.5825 \times 10^{-4}$ & $3.9999 \times 10^{-8}$ \\
0.3 & $2.8099 \times 10^{-4}$ & $6.0000 \times 10^{-10}$ \\
0.4 & $1.9751 \times 10^{-4}$ & $1.0999 \times 10^{-9}$ \\
0.5 & $4.0000 \times 10^{-4}$ & $5.8999 \times 10^{-8}$ \\
0.6 & $2.9448 \times 10^{-4}$ & $1.9200 \times 10^{-8}$ \\
0.7 & $5.4005 \times 10^{-3}$ & $3.7499 \times 10^{-8}$ \\
0.8 & $1.0297 \times 10^{-3}$ & $3.1899 \times 10^{-8}$ \\
0.9 & $1.3620 \times 10^{-3}$ & $5.8000 \times 10^{-9}$ \\
1.0 & $1.5920 \times 10^{-3}$ & 0 \\
\hline
\end{tabular}

in LWGM solution lesser than finite difference scheme implemented in Fermi the next generation CUDA computing architecture [13]. The method is also applied in moving boundary problems and provide good results. The boundary value problem of ordinary differential equation neither solved by variable coefficient method nor by power series method, it can be solved by the present method. The method can be successfully applied in non linear boundary value problems of ordinary and partial differential equations. The method can also be applied in one region or two region moving boundary value problems.

\section{Acknowledgements}

We are truly thankful to DST - CIMS and coordinator of DST - CIMS BHU VARANASI for proving necessary facilities.

\section{References}

[1] M.D. Mikhailov, Unified Analysis of Heat and Mass Transfer, A wiley- Interscience Publication, USA, 1983.

[2] K.N. Rai, S.K. Rai, Heat transfer inside the tissues with supplying vessel for the case when metabolic heat generation and blood perfusion are temperature dependent, Heat and Mass Transfer 35 (1999) 345-35.

Table 3: $\operatorname{LWGM}(9,1)$ and HGM solution with 12 degree Hermite polynomial [14].

\begin{tabular}{cccccc}
\hline $\mathrm{x}$ & Exact solution & HGM [14] & LWGM solution & AE $(\mathrm{HGM})$ & AE(LWGM) \\
\hline 0 & -0.2395322 & -0.2561473 & -0.2370244 & $1.6615 \times 10^{-2}$ & $2.5078 \times 10^{-3}$ \\
0.1 & -0.0903529 & -0.0878111 & -0.0915568 & $2.5418 \times 10^{-3}$ & $1.2038 \times 10^{-3}$ \\
0.2 & 0.2913156 & 0.2949467 & 0.2905488 & $3.6310 \times 10^{-3}$ & $7.6675 \times 10^{-4}$ \\
0.3 & 0.7311773 & 0.7350613 & 0.7310835 & $3.8839 \times 10^{-3}$ & $9.3886 \times 10^{-5}$ \\
0.4 & 1.0089780 & 1.0120826 & 1.0095671 & $3.1045 \times 10^{-3}$ & $5.8909 \times 10^{-4}$ \\
0.5 & 0.9399899 & 0.9412174 & 0.9410896 & $1.2274 \times 10^{-3}$ & $1.0997 \times 10^{-3}$ \\
0.6 & 0.4488151 & 0.4471576 & 0.4502001 & $1.6575 \times 10^{-3}$ & $1.3849 \times 10^{-3}$ \\
0.7 & -0.3895590 & -0.3948662 & -0.3882474 & $5.3071 \times 10^{-3}$ & $1.3116 \times 10^{-3}$ \\
0.8 & -1.3570362 & -1.3663642 & -1.3561420 & $9.3279 \times 10^{-2}$ & $8.9423 \times 10^{-4}$ \\
0.9 & -2.1489503 & -2.1621571 & -2.1486382 & $1.3206 \times 10^{-2}$ & $3.1216 \times 10^{-4}$ \\
1.0 & -2.4652569 & -2.4816165 & -2.4655925 & $1.6359 \times 10^{-2}$ & $3.3556 \times 10^{-5}$ \\
\hline
\end{tabular}


Table 4: Relative error (RE) in the present solution for different $M$.

\begin{tabular}{lcccc}
\hline $\mathrm{M}$ & 8 & 9 & 10 & 11 \\
\hline $\mathrm{RE}$ & $6.40401 \times 10^{-10}$ & $3.32959 \times 10^{-11}$ & $1.69948 \times 10^{-13}$ & $2.62657 \times 10^{-16}$ \\
\hline
\end{tabular}

Table 5: Execution time (ET) during in the process to determine $C$ and $y(x)$.

\begin{tabular}{lcccc}
\hline M & 8 & 9 & 10 & 11 \\
ET for $C$ & 0.000048 & 0.000048 & 0.000047 & 0.000111 \\
ET for $y(x)$ & 0.053188 & 0.069794 & 0.080037 & 0.230056 \\
\hline
\end{tabular}

Table 6: The effect of $S$ on $\operatorname{LWGM}(7,1)$ solution $\theta(x)$.

\begin{tabular}{ccccccc}
\hline- & LWGM & EM & LWGM & EM & LWGM & EM \\
\hline $\mathrm{x}$ & $S=1$ & $S=1$ & $S=3$ & $S=3$ & $S=5$ & $S=5$ \\
\hline 0.0 & 1.000000 & 1.000000 & 1.000000 & 1.000000 & 0.999997 & 1.000000 \\
0.1 & 0.887196 & 0.887195 & 0.872072 & 0.872068 & 0.862454 & 0.862437 \\
0.2 & 0.779256 & 0.779254 & 0.746260 & 0.746253 & 0.727954 & 0.727924 \\
0.3 & 0.665023 & 0.665020 & 0.624574 & 0.624564 & 0.599351 & 0.599309 \\
0.4 & 0.557302 & 0.557299 & 0.508826 & 0.508814 & 0.479112 & 0.479061 \\
0.5 & 0.452843 & 0.452839 & 0.400547 & 0.400534 & 0.369181 & 0.369129 \\
0.6 & 0.352321 & 0.352317 & 0.300929 & 0.300917 & 0.270902 & 0.270854 \\
0.7 & 0.256329 & 0.256326 & 0.210795 & 0.210785 & 0.184991 & 0.184950 \\
0.8 & 0.165364 & 0.165362 & 0.130592 & 0.130585 & 0.111554 & 0.111524 \\
0.9 & 0.079824 & 0.079822 & 0.060406 & 0.060401 & 0.050168 & 0.050155 \\
1.0 & 0.000000 & 0.000000 & 0.000000 & 0.000000 & 0.000000 & 0.000000 \\
\hline
\end{tabular}

Table 7: The effect of Legendre wavelets on $x_{f}$.

\begin{tabular}{lccccc}
\hline$(\mathrm{M}, \mathrm{k})$ & - & $(3,1)$ & $(5,1)$ & $(7,1)$ & - \\
\hline $\mathrm{S}$ & $F_{o}$ & LWGM $x_{f}$ & LWGM $x_{f}$ & LWGM $x_{f}$ & EM $x_{f}$ \\
\hline 1 & 0.1 & 0.402412 & 0.391964 & 0.392160 & 0.392160 \\
1 & 0.3 & 0.696999 & 0.678902 & 0.679242 & 0.679242 \\
1 & 0.5 & 0.899822 & 0.876459 & 0.876897 & 0.876897 \\
1 & 0.7 & 1.064683 & 1.037040 & 1.037559 & 1.037559 \\
1 & 1.0 & 1.272540 & 1.239500 & 1.240120 & 1.240120 \\
3 & 0.1 & 0.597531 & 0.577539 & 0.577906 & 0.577906 \\
3 & 0.3 & 1.034955 & 1.000328 & 1.000963 & 1.000963 \\
3 & 0.5 & 1.336121 & 1.291417 & 1.292238 & 1.292238 \\
3 & 0.7 & 1.580919 & 1.528026 & 1.528996 & 1.528996 \\
3 & 1.0 & 1.889560 & 1.826340 & 1.827500 & 1.827500 \\
5 & 0.1 & 0.688554 & 0.670700 & 0.670150 & 0.670213 \\
5 & 0.3 & 1.192611 & 1.161687 & 1.160734 & 1.160843 \\
5 & 0.5 & 1.539654 & 1.499731 & 1.498501 & 1.498642 \\
5 & 0.7 & 1.821744 & 1.774506 & 1.773050 & 1.773217 \\
5 & 1.0 & 2.177400 & 2.120940 & 2.119200 & 2.119400 \\
\hline
\end{tabular}


Table 8: The effect of Legendre wavelets on $\frac{d x_{f}}{d F_{o}}$.

\begin{tabular}{lccccc}
\hline$(\mathrm{M}, \mathrm{k})$ & - & $(3,1)$ & $(5,1)$ & $(7,1)$ & - \\
\hline $\mathrm{S}$ & $F_{o}$ & LWGM $\frac{d x_{f}}{d F_{o}}$ & LWGM $\frac{d x_{f}}{d F_{o}}$ & LWGM $\frac{d x_{f}}{d F_{o}}$ & EM $\frac{d x_{f}}{d F_{o}}$ \\
\hline 1 & 0.1 & 2.01206200 & 1.95982200 & 1.96080200 & 1.96080200 \\
1 & 0.3 & 1.16166500 & 1.13150400 & 1.13206900 & 1.132069000 \\
1 & 0.5 & 0.89982200 & 0.87645900 & 0.87689700 & 0.876897000 \\
1 & 0.7 & 0.76048800 & 0.74074300 & 0.74111300 & 0.741113000 \\
1 & 1.0 & 0.63627000 & 0.61975000 & 0.62006000 & 0.620060000 \\
3 & 0.1 & 2.98765700 & 2.88769700 & 2.88953100 & 2.889531000 \\
3 & 0.3 & 1.72492400 & 1.66721300 & 1.66827200 & 1.668272000 \\
3 & 0.5 & 1.33612100 & 1.29141700 & 1.29223800 & 1.292238000 \\
3 & 0.7 & 1.12922800 & 1.09144700 & 1.09214000 & 1.092214000 \\
3 & 1.0 & 0.94478000 & 0.91317000 & 0.91375000 & 0.913750000 \\
5 & 0.1 & 3.44277200 & 3.35350100 & 3.35074900 & 3.351066000 \\
5 & 0.3 & 1.98768500 & 1.93614400 & 1.93455600 & 1.934739000 \\
5 & 0.5 & 1.53965400 & 1.49973100 & 1.49850100 & 1.498642000 \\
5 & 0.7 & 1.30124500 & 1.26750400 & 1.26646400 & 1.266584000 \\
5 & 1.0 & 1.08870000 & 1.06047000 & 1.05960000 & 1.059700000 \\
\hline
\end{tabular}

Table 9: The effect of Legendre wavelets on $x_{f}$.

\begin{tabular}{lccccc}
\hline$(\mathrm{M}, \mathrm{k})$ & - & $(3,1)$ & $(5,1)$ & $(6,1)$ & - \\
\hline $\mathrm{S}$ & $F_{o}$ & LWGM $x_{f}$ & LWGM $x_{f}$ & LWGM $x_{f}$ & EM $x_{f}$ \\
\hline 1 & 0.1 & 0.459612 & 0.361328 & 0.361322 & 0.361322 \\
1 & 0.3 & 0.796071 & 0.625839 & 0.625828 & 0.625828 \\
1 & 0.5 & 1.027723 & 0.807954 & 0.807940 & 0.807940 \\
1 & 0.7 & 1.216018 & 0.955984 & 0.955968 & 0.955968 \\
1 & 1.0 & 1.378835 & 1.083985 & 1.083966 & 1.083966 \\
3 & 0.1 & 0.559852 & 0.509905 & 0.510075 & 0.510075 \\
3 & 0.3 & 0.969691 & 0.883181 & 0.883476 & 0.883476 \\
3 & 0.5 & 1.251866 & 1.140181 & 1.140563 & 1.140563 \\
3 & 0.7 & 1.481228 & 1.349081 & 1.349533 & 1.349533 \\
3 & 1.0 & 1.679555 & 1.529714 & 1.530226 & 1.530226 \\
5 & 0.1 & 0.586470 & 0.582922 & 0.582536 & 0.582536 \\
5 & 0.3 & 1.015795 & 1.009650 & 1.008982 & 1.009124 \\
5 & 0.5 & 1.311386 & 1.303452 & 1.302590 & 1.302774 \\
5 & 0.7 & 1.551653 & 1.542266 & 1.541245 & 1.541492 \\
5 & 1.0 & 1.759409 & 1.748765 & 1.747607 & 1.747854 \\
\hline
\end{tabular}

Table 10: The effect of Legendre wavelets on $\frac{d x_{f}}{d F_{o}}$.

\begin{tabular}{lccccc}
\hline$(\mathrm{M}, \mathrm{k})$ & - & $(3,1)$ & $(5,1)$ & $(6,1)$ & - \\
\hline $\mathrm{S}$ & $F_{o}$ & LWGM $\frac{d x_{f}}{d F_{o}}$ & LWGM $\frac{d x_{f}}{d F_{o}}$ & LWGM $\frac{d x_{f}}{d F_{o}}$ & EM $\frac{d x_{f}}{d F_{o}}$ \\
\hline 1 & 0.1 & 2.298059 & 1.806641 & 1.806609 & 1.806609 \\
1 & 0.3 & 1.326785 & 1.043065 & 1.043046 & 1.043046 \\
1 & 0.5 & 1.027723 & 0.807954 & 0.807940 & 0.807940 \\
1 & 0.7 & 0.868585 & 0.682846 & 0.682834 & 0.682834 \\
1 & 1.0 & 0.766020 & 0.602214 & 0.602203 & 0.602203 \\
3 & 0.1 & 2.799258 & 2.549523 & 2.550377 & 2.550377 \\
3 & 0.3 & 1.616152 & 1.471968 & 1.472461 & 1.472461 \\
3 & 0.5 & 1.251866 & 1.140181 & 1.140563 & 1.140563 \\
3 & 0.7 & 1.058020 & 0.963629 & 0.963952 & 0.963952 \\
3 & 1.0 & 0.933086 & 0.849841 & 0.850126 & 0.850126 \\
5 & 0.1 & 2.932348 & 2.914608 & 2.912679 & 2.913090 \\
5 & 0.3 & 1.692992 & 1.682750 & 1.681636 & 1.681873 \\
5 & 0.5 & 1.311386 & 1.303452 & 1.302590 & 1.302774 \\
5 & 0.7 & 1.108324 & 1.101618 & 1.100889 & 1.101045 \\
5 & 1.0 & 0.977449 & 0.971536 & 0.970893 & 0.971030 \\
\hline
\end{tabular}




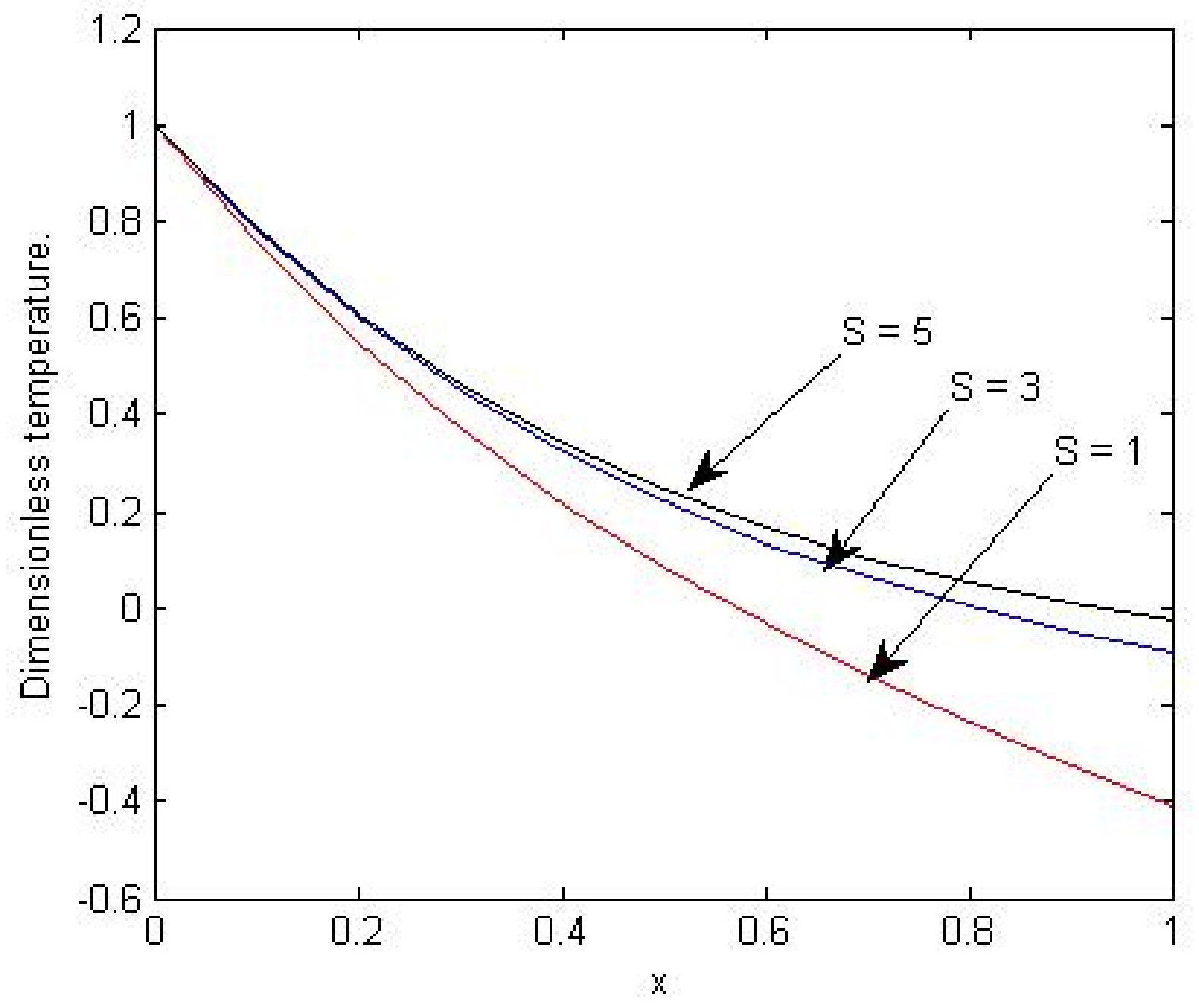

Figure 1: The effect of $x$ and Stafen number $S$ on the temperature. 


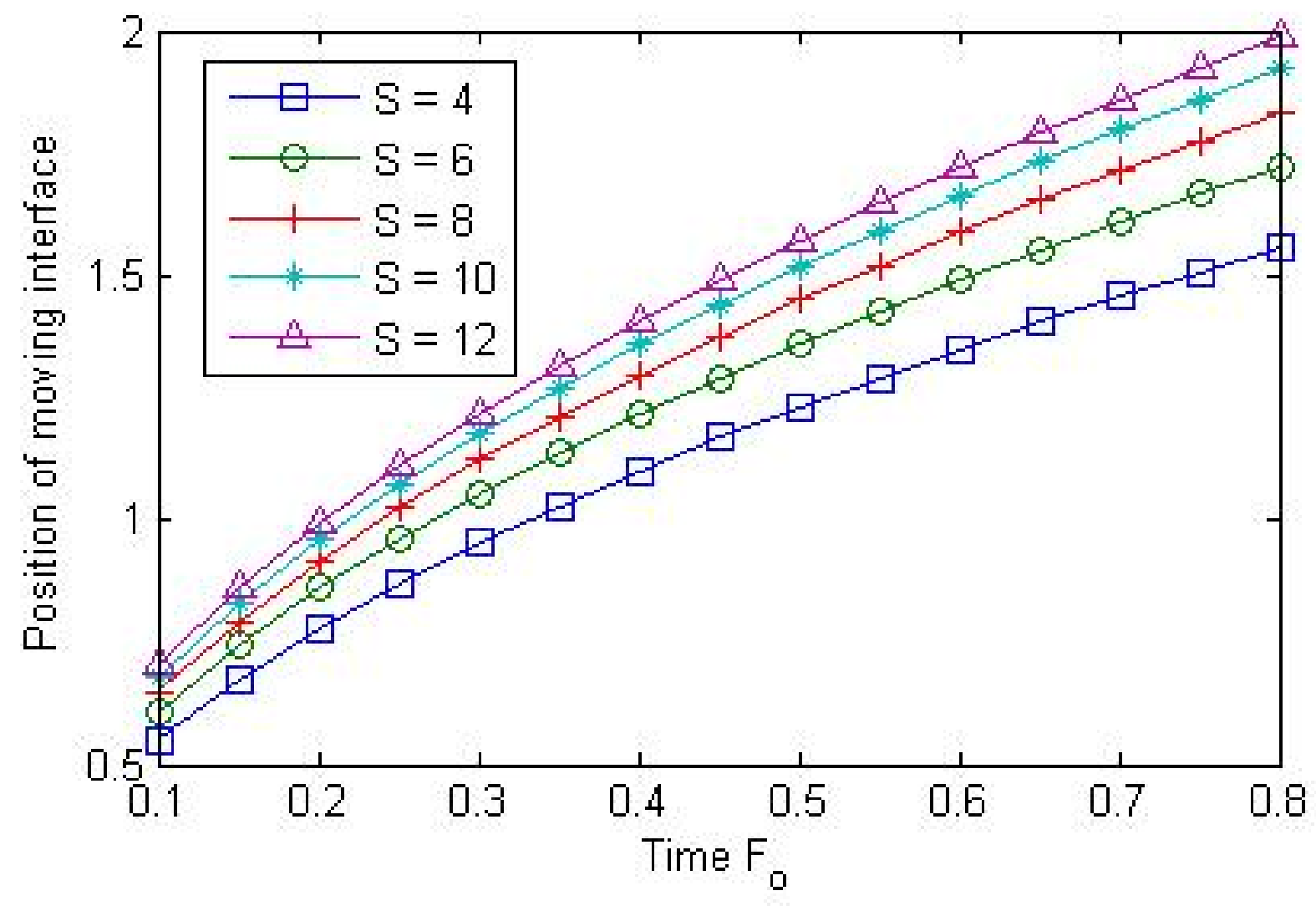

Figure 2: The effect of time $F_{o}$ and Stafen number $S$ on the position of moving interface $x_{f}$. 


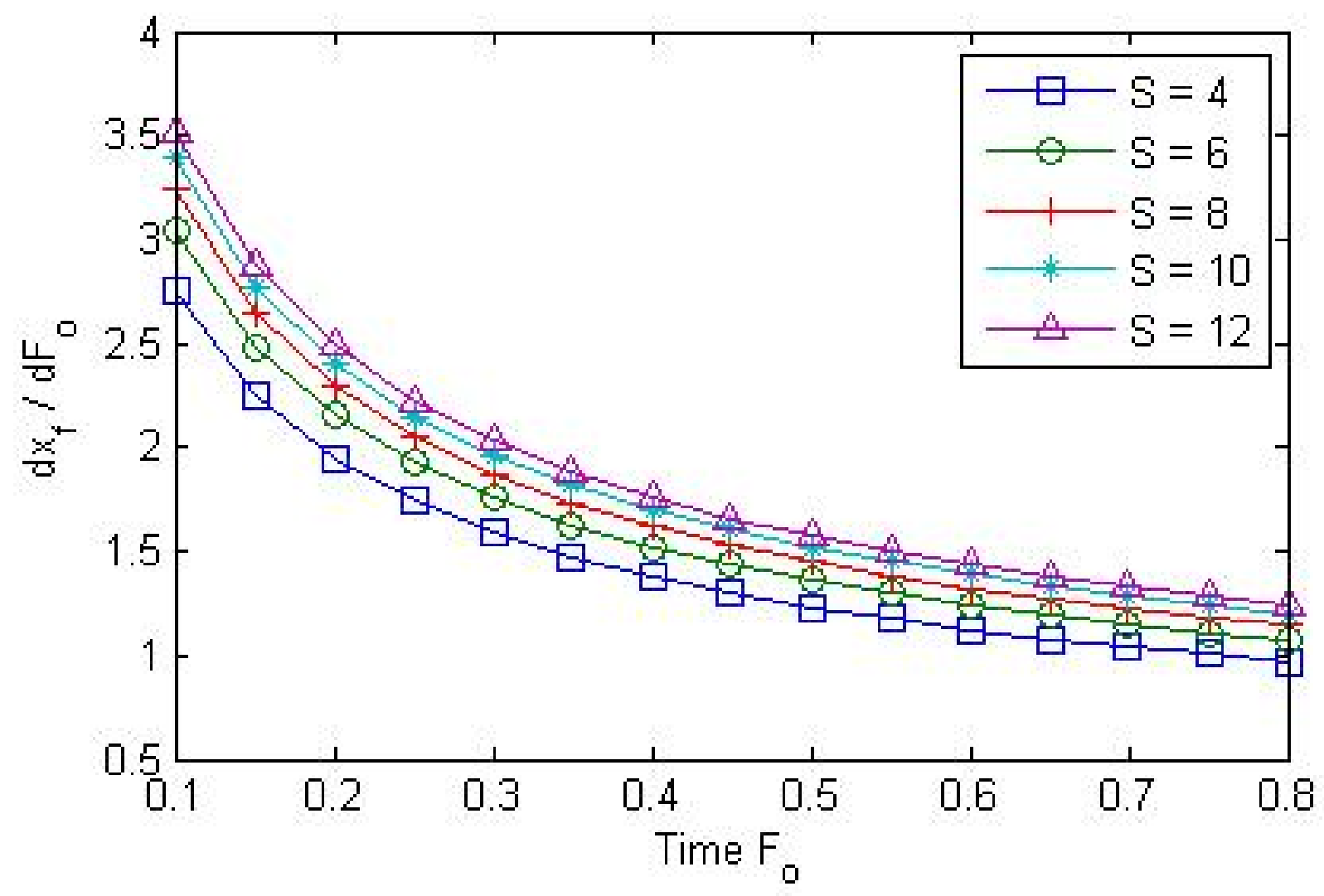

Figure 3: The effect of time $F_{o}$ and Stafen number $S$ on the rate of change of the position of moving interface with respect to time $\frac{d x_{f}}{d f_{o}}$. 
[3] C. Lanczos, Trigonometric interpolation of empirical and analytical functions, Journal of Mathematics and Physics 17 (1938) 123-129.

[4] C. Canuto, M. Hussaini, A. Quarteroni, and T. Zang, Spectral Methods in Fluid Dynamics, Berlin Springer 1988.

[5] B. Fornberg, A Practical Guide to Pseudospectral Methods, Cambridge, Cambridge University Press 1996.

[6] E. Babolian, M.M. Hosseini, A modified spectral method for numerical solution of ordinary differential equations with non-analytic solution, Applied Mathematics and Computation 132 (2002) 341-351.

[7] F. Mohammadi, M.M. Hosseini and S. T. Mohyud-Din, Legendre wavelet galerkin method for solving ordinary differential equations with non-analytic solution, Int. J. of Sys. 42 (2011) 579-585.

[8] M. W. Frazier, An introduction to Wavelets through Linear Alzebra, Springer, New York 1999.

[9] T. Lotfi, K. Mahdiani, Numerical solution of boundary value problem by using wavelet-galerkin method, Mathematical Science, 1, 3, (2007) 07-18.

[10] M. Razzaghi, S. Yousefi, Legendre wavelets operational matrix of integration, International Journal of Systems Sci. 32 (2001) 495-502.

[11] M. Yousefi, H. Derili Gherjalar and A. Arzhang, Numerical solution of the two point boundary value problems By using Wavelet bases of Hermite cubic spline wavelets, Australian Journal of Basic and Applied Sciences 5, 12, (2011) 2098-2105.

[12] K. Urban, Wavelet Method for Elliptic Partial Differential Equations, Oxford science publication, New York 2009 .

[13] P. Stpiczynski, J. Potiopa, Solving a kind of boundary-value problem for ordinary differential equations using Fermi the next generation CUDA computing architecture, Journal of Computational and Applied Mathematics, 236 (2011) 384-393.

[14] M. M. Rahman1, M.A. Hossen, M. Nurul Islam and Md. Shajib Ali1, Numerical solutions of second order boundary value problems by Galerkin method with Hermite polynomials, Annals of Pure and Applied Mathematics, 1, 2, (2012) 138-148.

[15] Steven C. Chapra, Applied Numerical Methods with MATLAB for Engineers and Scientists, Tata McGraw-Hill 2007.

[16] F. Keinert, Wavelets and Multiwavelets, Chapman and Hall/CRC, 2004.

[17] S. Upadhyay, K. N. Rai, Collocation method applied to unsteady flow of gas through a porous medium, 3 (3) (2014) 251-259.

[18] S. Singh, S. Upadhyay and K. N. Rai, Comparative analysis of power-law type fin problem using wavelet collocation and Galerkin methods, 3 (4) (2014) 534-546.

[19] S. Upadhyay, K. N. Rai, Wavelet collocation method applied to class of singular initial value problems arising in engineering, CONIAps XVII 2015. 